\title{
Protecting Networks with Intelligent Diodes
}

\author{
Jason Dahlstrom ${ }^{1}$ and Stephen Taylor ${ }^{2}$ \\ ${ }^{1}$ Web Sensing LLC, Lebanon NH, USA \\ ${ }^{2}$ Thayer School of Engineering, Dartmouth College, Hanover NH, USA \\ Jason.Dahlstrom@websensing.com \\ Stephen.Taylor@dartmouth.edu
}

\begin{abstract}
This paper explores the utility, practical nuances, performance characteristics, and attendant security risks associated with intelligent Diodes -- network appliances that regulate traffic flow by validating formats and mission payloads within the security perimeter afforded by a single Field Programmable Gate Array (FPGA). Diodes operate in the middleground between networks that are fully air-gapped -- i.e., completely disconnected from the Internet -- and those that are fully connected but require complex boundary defenses and security administration. As such, they provide an all-hardware, real-time alternative for protecting military vehicles and sensitive networks. Diodes are particularly useful in four core settings: when industrial plant need be connected to cloud-analytic services, such as Google-Analytics, for the purpose of process optimization; for supporting the lifecycle of military vehicles through Condition-based Maintenance; for preventing information bleed when sensor feeds must be consumed inside sensitive networks; and finally, for the reliable distributed replication of large files and databases. The devices may operate directly on protocol-specific traffic headers to dispatch or block traffic. Alternatively, they may be customized to validate file and traffic formats. This is achieved using an automated circuit design workflow that builds a hardware parsing plugin from a formal grammar and embeds it into the FPGA. This results in custom hardware that intelligently operates on mission specific data.
\end{abstract}

Keywords: data diode, google-analytics, condition-based maintenance, FPGA, cloud connectivity, data leak prevention

\section{Distribution}

This research is supported by the Defense Advanced Research Projects Agency (DARPA) under contract W31P4Q20-C-0033. The views, opinions and/or findings expressed are those of the authors and should not be interpreted as representing the official views of the Department of Defense or the U.S. Government. The paper is released with the following distribution determination:

Distribution A: (Approved for Public Release, Distribution Unlimited).

\section{Introduction}

Many organizations handle sensitive data and files relating to military missions, trade secrets, intellectual property, and/or private personal data (NBAR, 2021). Traditionally, these organizations have been well advised to implement an "airgap" that physically disconnects computers containing sensitive information from any connection to the Internet to protect against theft. Unfortunately, airgaps come with a substantial cost in productivity and maintenance as they assume that the relevant staff, with the expertise to handle network isolation, are co-located. Airgaps are also increasingly impractical given the need to connect critical systems, such as industrial plant, to cloud-based analytic platforms, such as Google Analytics, for the purpose of process optimization, or to support condition-based vehicle maintenance (Adams 2012, Carter 2013, Shanthakumaran 2010).

A technology alternative is the High Assurance Guard (HAG) (Heckman, Schell, and Reed 2012) often used in military cross-domain applications to share information between networks at different classification levels. These devices operate at the boundary between network segments to control and filter the flow of information; they often involve sophisticated virtual machine architectures and software stacks that must be supported by highly skilled network professionals with cumbersome incident reporting and investigative processes.

This paper considers an alternative Diode appliance, one instance of which is shown in Figure 1 (Dahlstrom and Taylor 2018, Aug 2019, Oct 2019, 2020, 2021). This device is marketed by Web Sensing LLC (websensing.com), a New Hampshire small business focussed on network security research. Internally, the device consists of a single custom circuit board (shown right). By design, the board employs a single FPGA chip (outlined) laying directly between the two leftmost Gigabit Ethernet (GigE) interfaces. The appliance thereby forms a "bump-in the-wire" between any pair of the network segments, with the FPGA forming the bridge for all communication. Consequently, it can monitor and interact with all systems attached to its interfaces and can act to store, analyze, 
and either forward or drop the traffic between them. It is important to note that outside the boundary of the FPGA, all the other circuitry is purely concerned with power and signal conditioning.
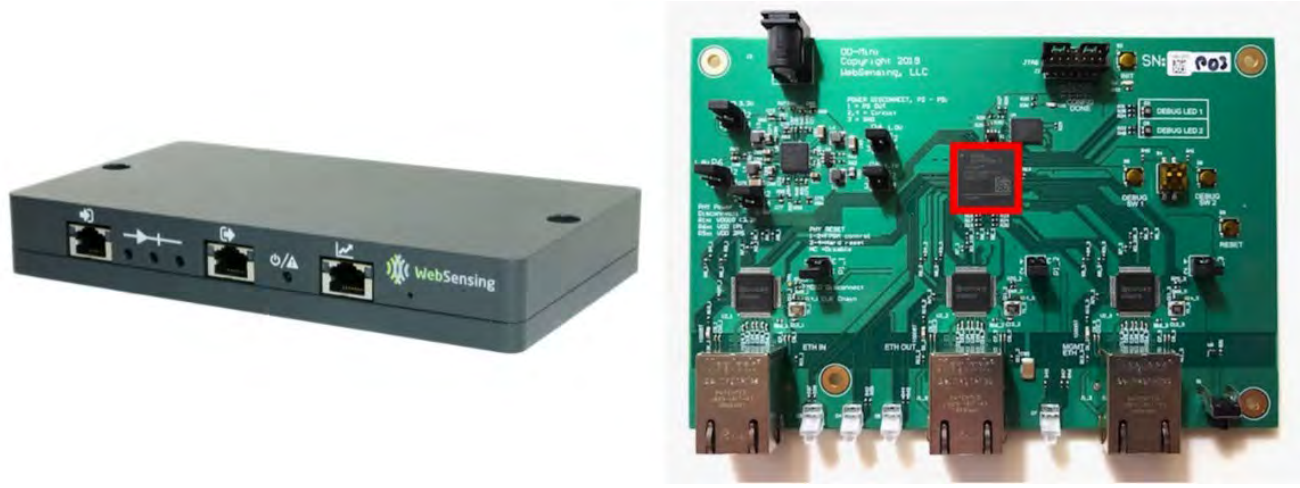

Figure 1: Diode Appliance

As an all-hardware appliance, the Diode offers several key security advantages: All sensitive data -- encryption keys, buses, and algorithmic functionality -- is strictly hidden within the security perimeter afforded by the FPGA chip-boundary, thereby mitigating reverse engineering in the event that a Diode is lost or stolen; No software is present in the device, thereby mitigating malicious implants and zero-day attacks and preventing its use as a steppingstone in sophisticated network attacks (Kushner 2013). An additional GigE connection (shown on the right) provides an isolated out-of-band channel that can be used to monitor the device or adapt its protection profile. Extensive anti-tamper techniques have been developed to enhance its resilience including the elimination of a JTAG interface and the ability to actively destroy circuits.

All the circuits resident in the Diode are developed using a rapid prototyping technology termed High Level Synthesis (HLS). This process is outlined in Figure 2 which contrasts software (blue), hardware (green) and HLS (brown) development pipelines.

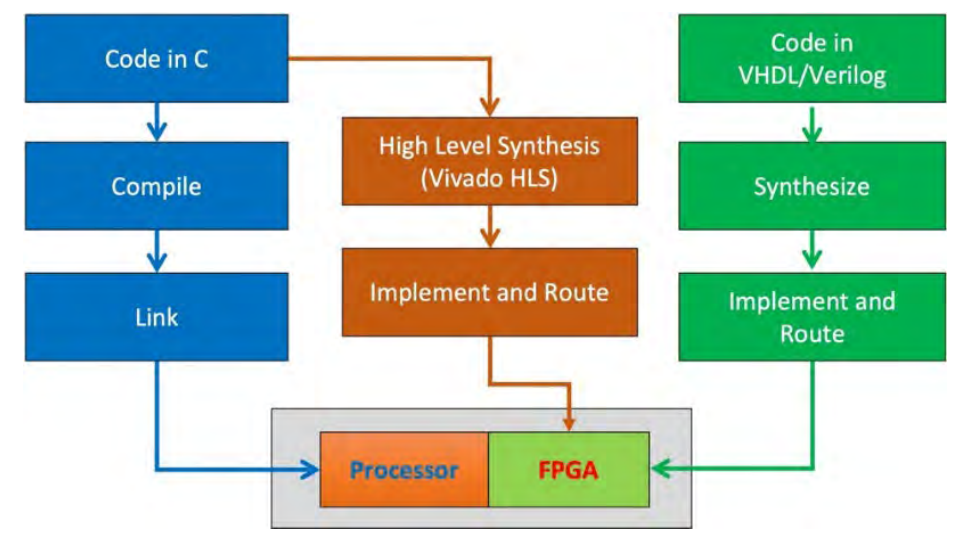

Figure 2: High-Level Synthesis

When generating software to run on a conventional processor, we generally develop code in some high-level language such as $\mathrm{C}, \mathrm{C}++$, or System- $\mathrm{C}$, then compile each module independently, link the resulting object files, and bootstrap the resulting binary image on the processor. Conversely, when developing for FPGA's, we design a circuit in a Hardware Definition Language (HDL) such as VHDL or Verilog, then synthesize it to produce a set of gates implementing the circuit definition, then implement and route the gates with standard FPGA circuit cells comprised of Flip-Flops, Lookup-tables, and blocks of Random-Access Memory. The result is a bit-file used to configure the FPGA circuit resources.

High-Level Synthesis provides an alternative route to configure an FPGA: The circuit operation is designed in software, then automatically converted into a synthesized circuit, which is then implemented and routed for the FPGA. The advantage of this approach is that an algorithm can be designed, along with associated testbench code, by any systems programmer with little specialize knowledge of hardware. The working software can then be automatically transformed into a standalone hardware block which is validated under co-simulation using the original software testbench. The validated block forms a reusable plugin that can be directly integrated into any static circuit design in the FPGA. Alternatively, the block can be treated as container, compliant with the 
Open Container Initiative (OCI) and akin to those used in Docker (c.f. docker.com). Using a technique known as partial reconfiguration, the FPGA can then be partitioned into compartments, or slots, linked together with industry standard Advanced eXtensible Interface (AXI) data-streams. Plugins can then be dynamically loaded into slots. To manage this process, a thin, all-hardware layer -- termed a Nanomarshal (Dahlstrom et al, Nov 2019) - has been developed whose operation is akin to a bare-metal hypervisor (Barham, et al 2003). A variety of pin-compatible FPGAs, with differing resources, are available for use on Diode appliances. The Artix-7 is the smallest family that supports partial reconfiguration; however, smaller Spartan-7 devices are available for static designs.

\section{Basic UDP Diode}

The conceptual floorplan for the simplest Diode circuit is shown in Figure 3; it provides one-way transport of only User Datagram Protocol (UDP) traffic. There are two separate GigE data paths: from the input port (IN) to the output port (OUT) for outbound traffic (green), and vice-versa for inbound traffic (blue). Interposed within these paths are two different packet inspection engine (PIE) plugins. The outbound PIE (green) inspects the packet headers on all arriving Ethernet frames, passes only the UDP-frames, and drops any others. The inbound PIE (blue) forms an open circuit by dropping all the arriving frames, thereby forming the Diode; though this block is wired into the printed circuit board, there are no connections within the PIE circuit itself providing the required isolation.

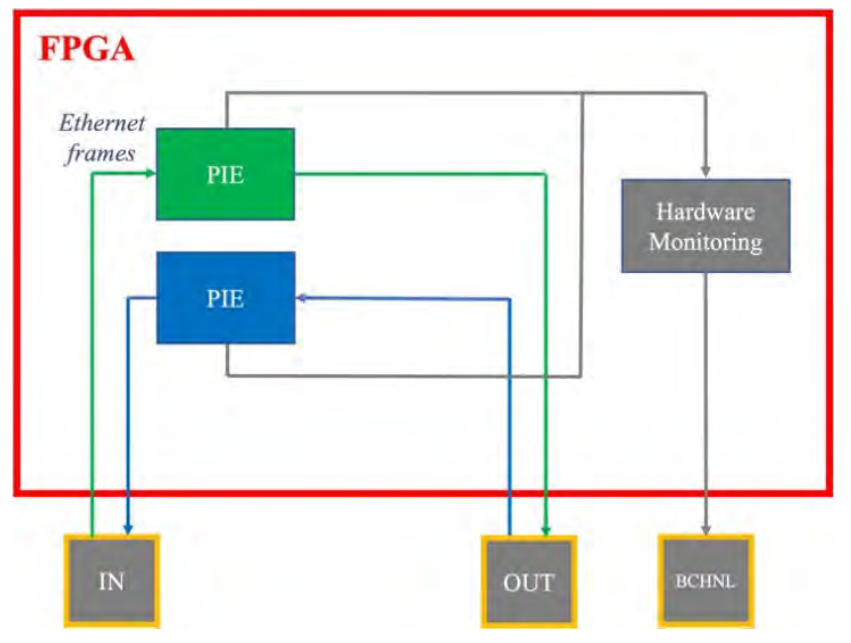

Figure 3: Diode Floorplan

The outbound PIE need only buffer a few bytes of the packet header to validate the protocol type; It can then either stream the remainder of the packet out of the device or drop it entirely. Consequently, the device achieves gigabit rates with sub-microsecond latency. Simple variations on this theme, that validate specific Ethernet MAC addresses, IP addresses, and/or UDP ports, can be added with negligible impact on performance.

Each PIE may accumulate statistical information concerning the number and type of packets being passed or dropped. This information can be made available on the backchannel periodically via a monitoring plugin that generates UDP or Simple Network Management Protocol Trap messages. These can be consumed by a standard Network Monitoring System (NMS), such as OpenNMS, Solarwinds, Nagios, Zabbix, etc. attached to the backchannel.

Since all inbound traffic is dropped, including Address Resolution Protocol (ARP) requests, it is necessary to manually configure ARP cache entries for devices attached to the Diode ports; this is a trivial exercise that can be achieved on Linux using the command:

sudo arp -s <IP_address $><M A C \_$address $>$

It is relatively trivial to add ARP validation to both PIE's and avoid the inconvenience, with a slight increase in risk and decrease in performance. 


\subsection{Use Case: Protecting High-value Assets and Condition-based Maintenance}

The core use case for the UDP Diode is to protect a single high-value asset connected directly to monitoring or analytics services, such as Google Analytics, for the purpose of process optimization as illustrated in Figure 4. Generally, these assets are designated systems whose compromise or damage would seriously impact corporate operations. Examples of such systems associated with industrial plant include laser and machine cutters; production-line assembly plant; HVAC and electrical supply systems; blast-furnace control systems; refinery control systems; waste and water treatment control systems; databases of production patterns, intellectual property, and facility details. In the medical arena, high-value assets include life-support systems, medical imagers, radiology equipment, and patient databases.

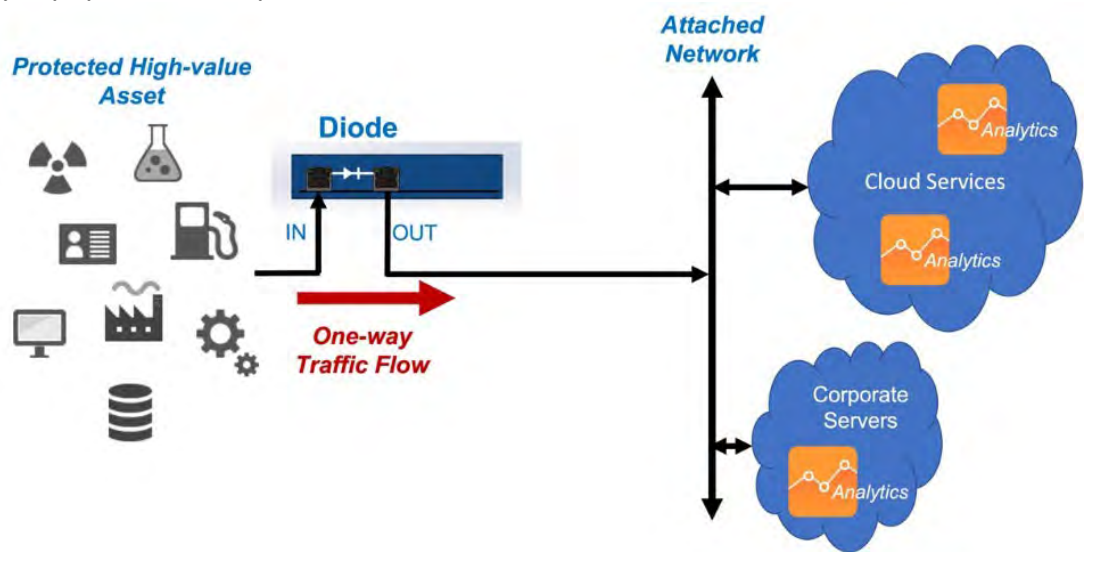

Figure 4: Protecting High-Value Assets

Since Diodes enforce one-way traffic in hardware, they provide isolation with a high degree of assurance against software attacks. To use a Diode in this manner it is co-located physically near the protected asset. The Ethernet cable at the asset is unplugged and attached to the Diode output; a short patch-cable is then connected from the asset to the Diode input. In this configuration, the asset may communicate status and performance data directly to analytics engines either at locally connected corporate servers, or alternatively, to cloud services located on the Internet. Since only one-way traffic flow is allowed, no traffic may pass from potentially compromised hosts downstream of the Diode either from within the organization or the Internet. This configuration is particularly useful when only periodic updates are required, or for periodically updating information between geographically distributed sites, such as corporate offices or hospitals.

The increasing prevalence of Condition-Based Maintenance (CBM) makes this configuration valuable for monitoring heavy vehicles, when combined in-line with wireless connectivity at the output port (OUT). The central idea is that vehicles are continuously monitored for indications of wear and failure with sensor data periodically uploaded to remote analytic servers. The analytics combine sensor data with historical component failure data and predictive models to determine when parts are likely to fail or need replacement, allowing replacements to be provided just-in-time, thereby reducing downtime, delays, and costs.

\subsection{Adding Encryption with Open-Source Software}

It is straight-forward to reliably transmit encrypted, signed files across a UDP Diode, then validate and decrypt them at a receiver. The Diode is directly connected between two servers: an inside or client server, located on a protected network behind the Diode, that serves up encrypted files and an outside server, that receives and decrypts the files as illustrated in Figure 5.

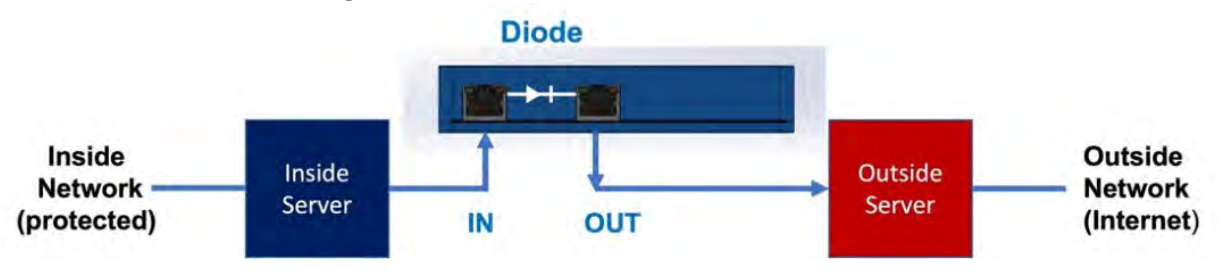

Figure 5: Encrypting Transfers

Each server must be equipped with the OpenSSL and UDPcast tools. OpenSSL provides a rich set of cryptographic tools for data privacy, authenticity, and integrity checking. OpenSSL's asymmetric encryption capabilities are also used to "sign" an encrypted file. The process generates a signature file that can be used to verify the 
authenticity of the sender as well as to validate the integrity of the file's contents. At the sender, it is used to encrypt and sign the file on the client (file sender) and then to verify and decrypt the file on the outside server (file recipient). UDPcast provides software capable of transferring data across a Diode. It provides data redundancy and data ordering in the one-way data stream necessary because the data receiver cannot alert the data sender of corrupted, missing, or out-of-order data.

Initially, a public/private key pair is generated for the outside server, for example, with the commands: openssl genrsa -aes256 -out server.private.key 8912

openssl rsa -in server.private.key -pubout -out server.public.key

The server.private.key is stored on the outside server and kept secret. The server.public.key is shared with anyone wishing to encrypt data intended for the outside server. As such, a copy of the server.public.key should be placed on the inside server. Similarly, a key pair for signing is generated on the inside server using the same commands - this time generating client.private.key and client.public.key files. The client.private.key is stored on the client server and kept secret. The client.public.key is shared with anyone wishing to verify authenticity and integrity attributes of flies signed by the client. As such, a copy of the client.public.key should be placed on the outside server. The sharing process for a file (e.g. message.txt) is then straightforward using the following steps:

1. Encrypt the file to transmit using the outside server's public key:

openssl rsautl -encrypt -pubin -inkey server.public.key -in message.txt -out encrypted.file

2. Sign the file to transmit using the inside servers private key: openssl dgst -sha512 -sign client.private.key -out encrypted.file.sha512 encrypted.file

3. Combine the encrypted file and its digest into a compressed archive bundle: tar -zcvf bundle.tgz encrypted.file encrypted.file.sha512

4. Start the udpcast server code on the outside server: udp-receiver --nokbd --receive-timeout 60 -f received.bundle.tgz

5. Send the bundle on the inside server using the updcast client code: udp-sender --async --max-bitrate $5 \mathrm{~m}$--fec $8 \times 8$-file bundle.tgz

6. Uncompress and unpack the compressed archive file on the outside server: tar -zxvf received.bundle.tgz

7. Verify the authenticity of the bundle's sender and the bundle's file integrity: openssl dgst -sha512 -verify client.public.key -signature encrypted.file.sha512 encrypted.file

8. Decrypt the encrypted file:

openssl rsautl -decrypt -inkey server.private.key -in encrypted.file -out message.txt

This entire process can easily be automated with simple shell scripts for Diode users.

Although the two servers could be anywhere, to simplify the process we have developed a complete Gateway appliance, shown in Figure 6. This system combines the Diode with two Next Unit of Computing (NUC) computers (top left and right) that act as the designated servers, running either Windows or Linux. This system also includes isolated power supplies, associated with the inside and outside networks, to protect against power-line exploitation across the Diode. Each server has two network interfaces: one to the Diode, and the other to either the inside or outside networks. Since the network connections to the Diode are direct, they are extremely lowloss making transport reliable.

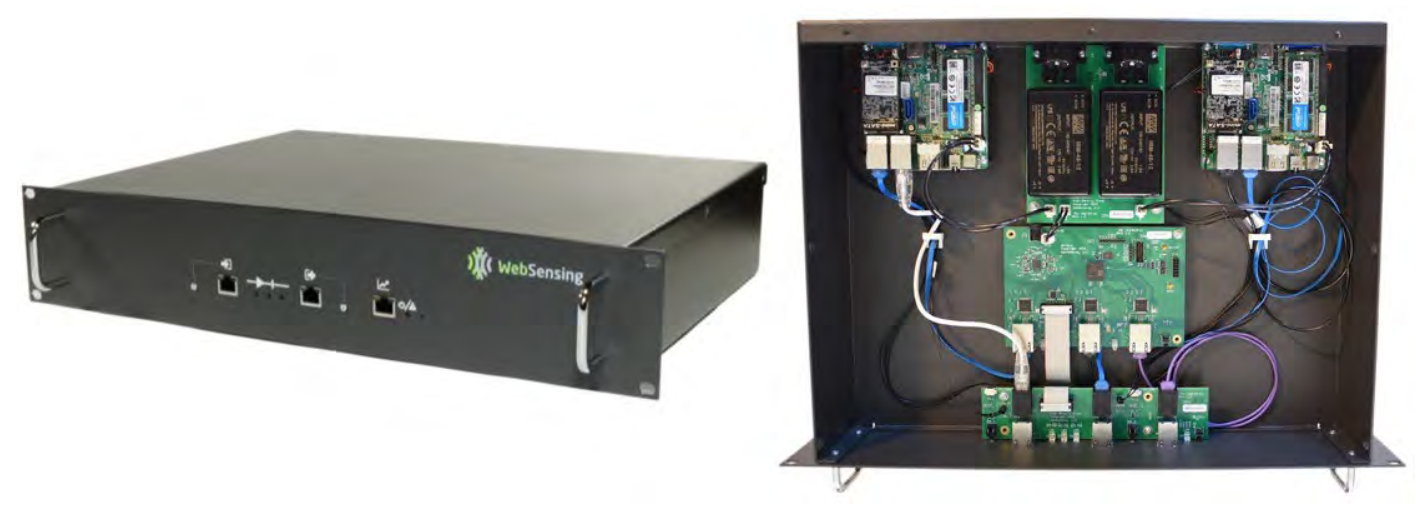

Figure 6: Gateway Appliance 


\subsection{Use Case: Low-value Assets and Database Replication}

Many industries maintain large files and databases associated with their core industrial processes to track and optimize production or billing, and for historical purposes. Some organizations also require the ability to securely copy them between geographically dispersed locations. Thus, some applications may have stringent reliability concerns that require the guarantee that all data transitioning a Diode is successfully received.

Turnstile technology is a patented hardware mechanism that provides reliable one-way transfers without violating the one-way data flow guarantees provided by a Diode (Dahlstrom and Taylor, 2021). The technology is fully contained within the FPGA circuit design shown in Figure 3 and thus can be used with either Diode or Gateway appliances. The essential idea is that the sending computer or server wraps its transmissions with size and sequencing information. The receiver generates an acknowledgement upon successful completion of a transfer, that is intercepted on the inbound link at the Diode. Hardware within the Diode internally generates a completely isolated response to the sender indicating the successful completion of the transfer. This occurs without transferring any information from the output to the input of the Diode, ensuring that no network-borne exploits can transition the Diode.

There are often non-essential or support assets within an organization that serve ancillary engineering and administrative services, such as storage and manipulation of intellectual property, payroll, or personal data. Elements of each individual service constantly exchange information and the interposition of individual Diodes, as shown in Figure 4, would cause unacceptable interference in normal operations. Unfortunately, these lowervalue systems can serve as an easy target if unprotected, allowing theft of intellectual property, or simply acting as a foothold within the organization for information gathering or launching network attacks later. In such instances, it is preferable to protect independent segments of the Local Area Network (LAN) that contain a set of related systems or databases as shown in Figure 7: Here a single Diode protects an entire LAN segment -- with all its workstations, systems, databases, and servers - allowing them to inter-operate freely as a single entity. Turnstile technology allows reliable controlled transfers between such segments, akin to air-gapping them.

Typically, LAN's are organized with a collection of rack mounted network switches and routers placed in routing closets. Individual segments are connected into the switching infrastructure through standard patch-panels allowing for flexible reconfiguration. The Diode and Gateway appliances can thus be easily inserted between segments, simply by moving cables.

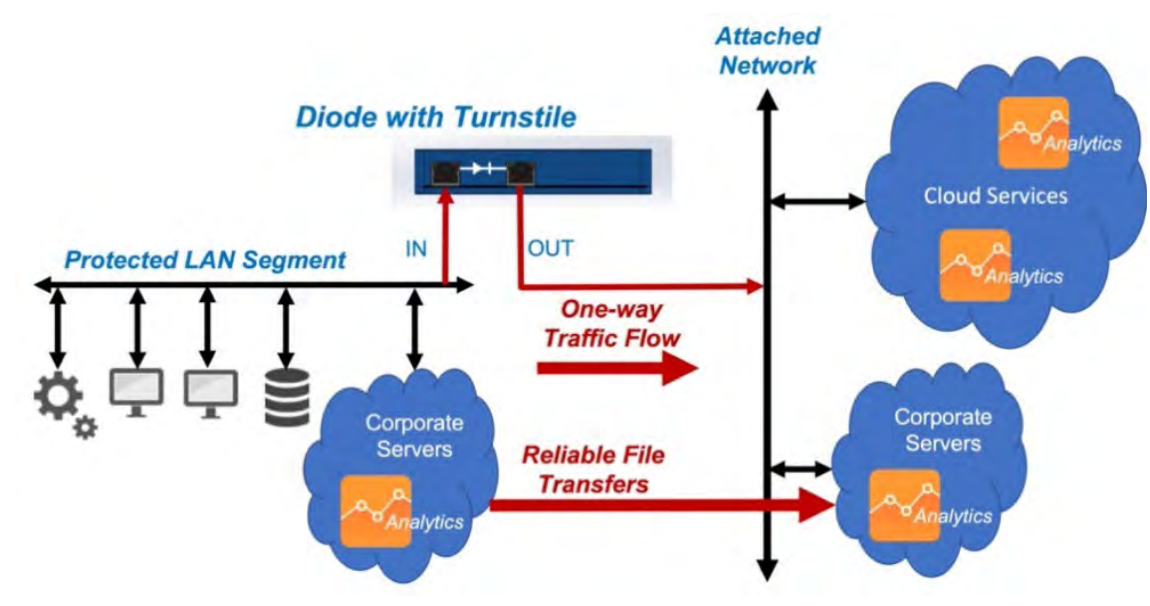

Figure 7: Reliable Transfers

\section{Building Diode Intelligence}

Figure 8 refines Figure 4 and provides the conceptual floorplan of an intelligent Diode, illustrating typical flows and plugin blocks that can be inserted within the FPGA either statically or dynamically. As before, there are two basic data paths: from the input port (IN) to the output port (OUT) for outgoing traffic, and vice-versa for incoming traffic. 


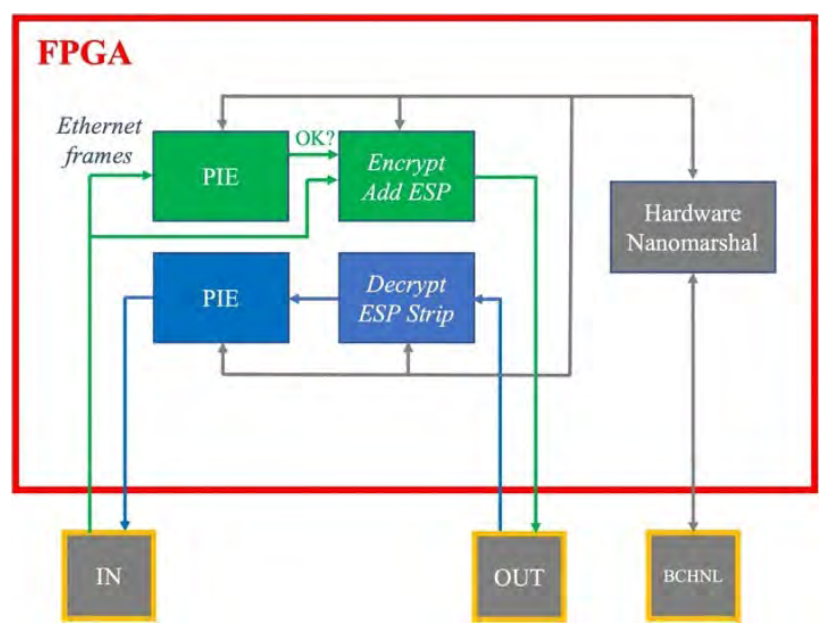

Figure 8: Intelligent Diode Floorplan

The green path in Figure 3 illustrates a typical outbound flow that concurrently inspects, encrypts, and encapsulates Ethernet frames completely in hardware. If the packet inspection algorithm determines that a frame is valid -- in terms of its protocol headers and payload content -- it signals (c.f. OK) the downstream blocks causing them to transmit the encrypted frame within an encapsulation payload; otherwise, the encrypted frame is discarded. Similarly, a typical inbound flow, shown in blue, might strip encapsulation and decrypt a frame, then validate the inbound frame and either pass or drop it.

Each of these blocks can be replaced by the degenerative case of a wire, that passes all traffic, or an open circuit, that drops all traffic. Consequently, a wide variety of appliances can be realized with the same basic structure. For example, the simple User Datagram Protocol (UDP) Diode discussed previously is composed of a green path that checks the IP protocol header to ensure each frame is UDP; the encryption/encapsulation block is replaced by a wire, and the inbound path by an open circuit. Thus, only valid UDP packets pass from input to output, and all inbound traffic is dropped. Alternatively, a Transport Control Protocol (TCP) Diode involves an inbound path that passes only valid connection requests and data packets, and an outbound path that allows only valid TCP flow synchronization packets.

The third (rightmost) GigE port providing the backchannel in Figure 8, serves to provide a direct connection to Nanomarshal hardware allowing plugins to be dynamically installed within the dataflow of the device; alternatively, it may be populated by a simple monitoring block used Figure 4 .

\subsection{Hardware Encryption Performance}

The appliance shown if Figure 8 may leverage any encryption and/or encapsulation scheme rendered into a plugin via HLS. By default, an AES-256 plugin, certified using the NIST Cryptographic Algorithm Verification Program (CAVP) is available, with the standard Encapsulating Security Payload (ESP).

It is possible to render the AES algorithm into a wide variety of alternative implementations using pragmas that inform HLS how to layout and route the attendance circuits. This results in a trade-space that allows circuit performance -- bandwidth, maximum clock frequency, and latency - to be successively traded for FPGA resources -- Block RAM, Flip-flops, and Lookup-Tables.

Benchmark studies led to the discovery of a "sweet spot" in this trade-space that achieves close to Gigabit line speed -- $0.82 \mathrm{~Gb} / \mathrm{sec}--$ with only $2.5 \%$ LUT utilization on an Artix 200T FPGA device. Note that generally two instances of the encryption block are required: one for encryption and one for concurrent decryption, with similar resource requirements. Though it is possible to achieve $1.1 \mathrm{~Gb} / \mathrm{sec}$ - exceeding line speed - this comes at heavy cost in LUT recourses: 20\% of the Artix 200T FPGA per instance. Unfortunately, the AES Cyclic Block Chaining (CBC) mode of operation has a data dependency that limits pipelining. However, Counter mode relieves this dependency and yields performance approaching $10 \mathrm{~Gb} / \mathrm{sec}$.

\subsection{General Packet Inspection and its Performance}

The typical action of a Diode on detecting a valid message is to allow it to pass; conversely, its action on detecting invalid data is to drop the message, thereby mitigating potential exploitation, but may also involve generating 
an alert on the backchannel. Complex validation tasks, beyond the protocol header checks outlined in Section 3 , might involve sophisticated tests to ensure that the messages adhere safely to industry standard protocols, that message fields contain only legitimate values, and/or that message content is valid within the context of a particular mission.

In general, parsing is the process of validating an input stream of symbols by understanding their format (syntax) and meaning (semantics). For example, compilers such as GCC use a parser to validate that a computer program, written in some programming language such as $\mathrm{C} / \mathrm{C}++/ J a v a / F o r t r a n$ is written correctly (i.e. is syntactically valid), and to understand its structure (i.e. its semantics) for the purpose of machine code generation and optimization. Parsers are tools that apply a collection of formal grammar rules, defining some input language, to determine if the input adheres to the rules. For example, the following 3-rule grammar $\mathrm{G}$ defines a language in which a stream of symbols is valid only if it begins with the character ' $a$ ', ends with ' $c$ ', and contains one or more intervening ' $b$ ' characters:

$\mathrm{G}:$ ' $\mathrm{a}$ ' $\mathrm{Bs}$ ' $c$ ' ;

Bs: 'b' | Bs 'b' ;

The "or" symbol | designates an alternative definition for the rule defining "Bs". This grammar accepts as valid the input streams $a b c, a b b c$ and $a b b b c$ etc, but rejects any other stream, e.g. $a, a c, a a a, c c c, a d x, a b b b x$, etc. Individual characters such as ' $a$ ' are terminal symbols that must be present in the input stream; all other symbols are non-terminals representing intermediate structural elements. For binary grammars, hexadecimal terminal values can also be used (e.g., 'XXF' represents a single byte value corresponding to 255 in decimal).

Parser generators are tools that take a grammar as input and automatically generate a program that implements the associated parser. The most mature and widely used generator is Bison which accepts two primary classes of grammar: Generalized Look-Ahead (GLR) and the more restrictive Left-to-Right Look Ahead (LALR). Both classes of grammar are expressed in Backus-Naur Form (BNF), used above to define the grammar G. Under the DARPA SafeDocs program, new tools are being developed based on formal methods. One of the most mature is the Hammer combinator library which provides a collection of well-defined base parsers and methods to combine them to build more complex parsers. The resulting parsers are provably correct by construction. The Hammer library provides a collection of backends that allow different classes of grammar to be implemented, including GLR and LALR.

Though GLR grammars are more general, LALR grammars are sufficient for validating a wide variety of protocol and file formats, where the input is in either ASCII and/or binary. These grammars can be realized with a generic push-down automaton - a finite state machine employing a single stack to store symbols while parsing the input stream. The state machine relies on two core operations shift - involving saving a symbol from the input onto the stack and reduce - involving the application of a grammar rule to detect a structure in the input and reduce the symbols on the stack. The state-machine is generic and common to all grammars, however, the order in which shift and reduce operations are applied to the input is based on a collection of parsing tables derived from the grammar, usually referred to as Action/Goto tables (Aho, Sethi, and Ullman, 1988). These tables map the current state of the automaton, to a next state based on the symbol read from the input stream.

A completely automated process has been developed that takes an LALR grammar, such as G above -- written in either Bison or Hammer -- and produces a validated parser plugin that can be inserted into a Diode as a packet inspection engine (PIE). This process involves the automatic generation of an optimized set of parsing tables, their combination with generic automaton code, and a translation process based on HLS. The primary use of FPGA resources in the resulting parsers is BRAM used to store the parsing tables. For concreteness, consider the full JSON grammar (c.f. json.org). In Bison, it consists of approximately 325 rules and its corresponding state machine has 689 states. The associated implementation requires $1.46 \mathrm{Mbytes}$ of BRAM resources to implement, which corresponds to $\sim 2 \%$ of the BRAM resource on an Artix 200T device; a negligible percentage of other resources is also required.

Parsing is inherently a byte-by-byte process that builds knowledge of the structure of the input stream seen up to a particular point. In consequence, the time to parse a message or file is directly proportional to the length of the input. For the above JSON grammar, the initial prototype has a processing time of approximately 45 clock cycles per byte; at $125 \mathrm{MHz}$ the time required to process an Ethernet frame (1600 bytes) is 575 microseconds. Though the link may operate at Gigabit rates, when parsing the bandwidth drops -- by virtue of the need to 
store, parse, and forward individual Ethernet frames sequentially -- generally varying between 2.25 and 3.5 $\mathrm{Mb} / \mathrm{sec}$. All our optimization efforts thus far have focussed on reducing FPGA resource requirements, to ensure that the parsers can fit into the FPGA's, rather than latency and bandwidth. Currently, the parsers constructed automatically are only able to validate syntax. For semantic checks, they are augmented with hand-coded Cfunctions prior to HLS, a process we expect to mature over time.

\subsection{Use Case: Preventing Network Bleed}

Generally, the perception of a Diode is that it protects assets from inbound attacks. However, it is important to understand that there is a reciprocal value when a Diode is configured in the reverse direction. Figure 9 shows this alternative with a Diode connected such that its input (IN) is connected to the outside network, while its output (OUT) is connected to the protected network. This configuration has the objective of allowing sensor feeds and database transfers from untrusted networks into the protected network, while ensuring that no data can exit the protected network, i.e., that no valuable information can escape, or bleed out of it.

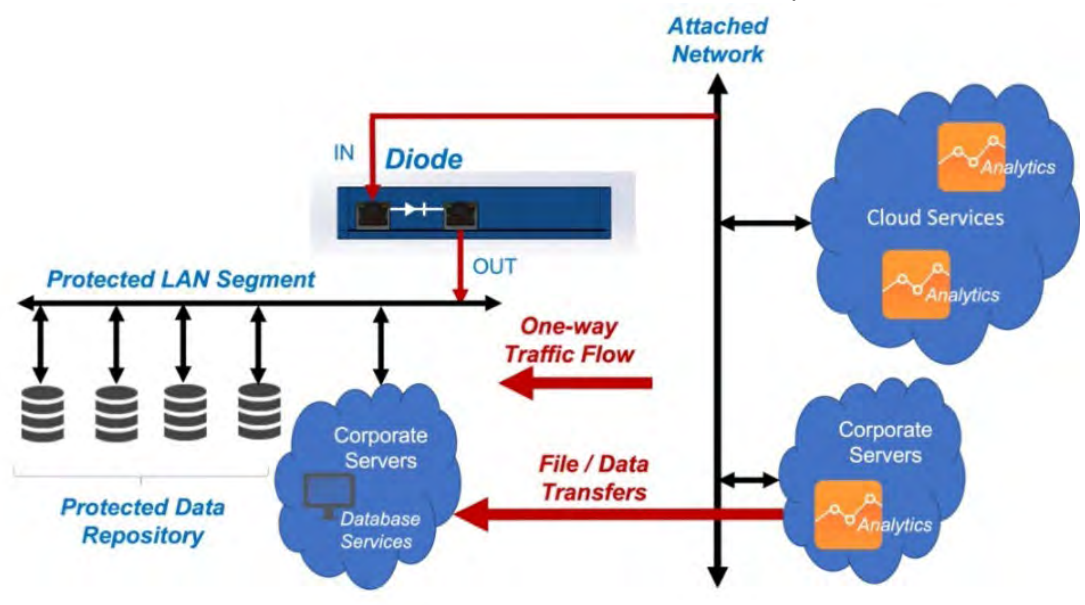

Figure 9: Preventing Information Bleed

Clearly the value of timely data collection must be weighed against the attendant risks associated with this approach. However, the generalized Diode offers the opportunity to insert highly specialized mission- and timedependent validation into data streams to mitigate these risks.

\section{In Conclusion}

This paper has presented a methodology for building a wide variety of intelligent Diode appliances with their attendant use cases. The methodology is based on a collection of hardware plugins that can be statically configured or dynamically installed, to adapt to a perceived threat level, via an out-of-band channel. At one extreme the appliances use protocol header information to validate traffic in real-time. At the other extreme, sophisticated intelligence is built into them using automation techniques. This intelligence is capable of carefully checking every byte in a transfer to validate detailed mission-specific payloads. The use of multiple Diodes within a corporate environment allows network administrators to carefully control and shape the available attack surface to protect valuable assets. This provides precise control of what machines are accessible, from where, and what data is allowed to flow, in which direction between them.

\section{Acknowledgements}

The authors wish to thank and acknowledge the work of Stephen Padnos, who designed the Diode hardware, and Jim Brock who designed the automation workflows.

\section{References}

Adams,C. (2012) "HUMS Technology", Aviation Today (aviationtoday.com/2012/05/01/hums-technology).

Aho, A.V., Sethi, R., Ullman,. J.D. (1988) Compilers, Addison Wesley.

Barham P, et al (2003) "Xen and the Art of Virtualization", In: Proceedings of the nineteenth ACM symposium on Operating systems principles, pp 164-177.

Carter, M.C. (2003) “Post Implementation CBM Benefit Analysis”, Annual Conference of the Prognostics and Health Management Society.

Dahlstrom, J., and Taylor, S. (2018) "System-on-Chip Data Security Appliance and Methods of Operating the Same", U.S. Patent $10,148,761$. 


\section{Jason Dahlstrom and Stephen Taylor}

Dahlstrom, J., and Taylor, S. (Aug 2019) "System-on-Chip Data Security Appliance and Methods of Operating the Same", U.S. Patent $10,389,817$.

Dahlstrom, J., and Taylor, S. (Oct 2019) "Endpoints for Performing Distributed Sensing and Control and Methods of Operating the Same", US Patent 10,440,121.

Dahlstrom, J., et al, (Nov 2019) "Hardening Containers for Cross-Domain Applications", In Proceeding of MILCOM 2019, Norfolk, VA, USA, pp. 1-6.

Dahlstrom, J., and Taylor, S. (2020) "System-On-Chip Data Security Appliance Encryption Device and Methods of Operating the Same" U.S. Patent 10,616,344.

Dahlstrom, J., and Taylor, S. (2021) “Hardware Turnstile”, U.S. Patent 10,938,913.

Goldberg R.P. (1974) "A survey of virtual machine research", In: Proceedings of Computer, 7th edn., pp 34-45.

Heckman, M.R., Schell, R.R., and Reed, E.E. (Dec 2012) "Toward formal evaluation of a high-assurance guard”, In:

Proceedings of the $6^{\text {th }}$ Layered Assurance Workship (LAW) 2012, December 2012, Orlando, FL. pp. 25-29.

Kushner, D. (2013) "The Real Story of STUXNET", IEEE Spectrum.

NBAR (2021) -- The National Bureau of Asian Research, The IP Commission Report.

Shanthakumaran, P. (2010) "Usage Based Fatigue Damage Calculation for AH-64 Apache Dynamic Components", The American Helicopter Society 66th Annual Forum, Phoenix, Arizona. 